\title{
Coupled Photon and Heat Transport Simulation inside Biological Tissue for Laser Therapy*
}

\author{
Atsushi SAKURAI**, Isami NITTA**, Shigenao MARUYAMA***, \\ Junnosuke OKAJIMA*** and Koji MATSUBARA** \\ ** Department of Mechanical and Production Engineering, Niigata University \\ 8050 2-no-cho Ikarashi, Nishi-ku, Niigata, Japan \\ E-mail:sakurai@eng.niigata-u.ac.jp \\ *** Institute of Fluid Science, Tohoku University \\ 2-1-1 Katahira, Aoba-ku, Sendai, Japan
}

\begin{abstract}
The objective of this study is to develop a new simulator for laser therapy. In order to minimize undesired effects on the laser therapy, it is important to precisely predict for heat and photon transport inside a biological tissue. In the present study, the photon transport is solved with the Radiation Element Method by Ray Emission Model $\left(\mathrm{REM}^{2}\right)$, and then coupled with bioheat transfer equation. The dimensionless results become valuable guidance for developing a laser therapy system.
\end{abstract}

Key words: Laser, Heat Conduction, $\mathrm{REM}^{2}$, Biomedical Application

\section{Introduction}

Laser therapy for a skin disease such as a mole or a macula is a promising technology due to their convenience and the fewer side-effects ${ }^{(1-2)}$. One of the principles of Laser therapy is that thermal energy converted from laser in biological tissue treats the target disease. This principle is also called a hyperthermia ${ }^{(3)}$. This treatment is not limited to a skin disease but also effective for a tumor, because, it is well known that a tumor can be killed by heat. However, laser therapy includes problem that it is quite difficult to measure a heat and light energy transport inside a biological tissue. Optical tomography is one of the promising techniques to visualize these phenomena ${ }^{(4)}$. This imaging technique has necessary to develop an advanced reconstruction algorithm for a photon transport. Therefore, laser therapy possesses an element of risk that a healthy tissue may be damaged by the excess heat energy. Furthermore, effects of optical and thermophysical properties of biological tissue for the coupling phenomena have not been understood well.

For the reason described above, it is important to precisely predict and understand heat and photon transport phenomena inside a biological tissue. A fast and accurate coupled photon and bioheat transfer simulation model is required to minimize undesired effects on the laser therapy, but that kind of simulation model has not been established satisfactory.

The radiative transfer equation (RTE) has successfully been recognized as a widely-used optics model for describing photon transport in biological tissue. Photon propagation models in biomedical optics are essential for optical coherent tomographic imaging using visible and near-infrared light. Since the biological tissue is a strong scattering media, Monte Carlo photon transport simulation has been used as effective tool for this research area ${ }^{(5-6)}$. However, Monte Carlo method is generally time-consuming method due to the stochastic approach.

In the present study, the photon transport is solved with the Radiation Element Method by Ray Emission Model $\left(\mathrm{REM}^{2}\right)^{(7-9)}$. The REM ${ }^{2}$ employs a deterministic approach so that the computational speed is faster than Monte Carlo method. In order to describe a photon 


\section{Nomenclature}

$a_{l} \quad$ : forward scattering parameter

$A_{i}^{R} \quad$ : effective radiation area

$c_{p} \quad$ : specific heat

$E_{b} \quad$ : black-body emissive power

$F_{i, j}^{A} \quad$ : absorption view factor

$F_{i, j} \quad$ : diffuse scattering view factor

$F_{i, j}^{E} \quad$ : extinction view factor

$f \quad$ : fraction of energy

I : intensity

$k \quad$ : thermal conductivity, [W/mK]

$\beta \quad$ : extinction coefficient

$\varepsilon_{j} \quad$ : emissivity

$\mu \quad$ : direction cosine

$\rho:$ density

$\gamma:$ blood perfusion rate, $\left[\mathrm{m}^{3} /\left(\mathrm{s} \cdot \mathrm{m}^{3}\right)\right]$

met : metabolic

$D \quad$ : zeroth-order delta function approximation

$T \quad$ : total
$N \quad$ : conduction-radiation parameter

$L \quad$ : number of discrete direction

$q \quad$ : heat flux

$Q_{R, j} \quad$ : radiant energy for element $\mathrm{j}$

$t \quad$ : time

$T$ : temperature

$V \quad$ : volume

$X \quad$ : maximum depth

$x \quad$ : space variable

$\Psi \quad$ : dimensionless heat flux

$\theta \quad:$ dimensionless temperature

$\sigma \quad$ : Stefan-Boltzmann constant

$\xi \quad:$ dimensionless time

$\omega \quad$ : scattering albedo

$C \quad$ : conductive

$R \quad$ : radiative

* : dimensionless

and heat transport phenomena in biological tissue, the photon transport model coupled with bioheat transfer equation. We provide dimensionless results that become valuable guidance for developing a laser therapy system. The first stage of this research involves that continuous-wave laser therapy simulation is performed in a standard homogeneous skin model. The constructed simulation model will be extended for pulsed laser in real disease situations in the future study. This fundamental study is useful for not only laser therapy but also radiation heating therapy, biomedical optics, etc.

\section{Formulation}

The bioheat transfer equation ${ }^{(10)}$ coupled with photon transport is described as follows,

$$
\rho c_{p} \frac{\partial T}{\partial t}=k \frac{\partial}{\partial x}\left(\frac{\partial q_{c}}{\partial x}\right)+q_{m e t}+\rho_{b} c_{b} \gamma_{b}\left(T_{r e f}-T\right)-\frac{\partial q_{R}}{\partial x},
$$

where, $\rho$ is the density, $c_{p}$ is the specific heat, $q_{m e t}$ is the metabolic heat production, third term of right-hand side is the blood perfusion term and $q_{R}$ is the heat flux converted from laser energy. When a laser irradiates a biological tissue, photon and heat propagate three-dimensionally from the laser spot. In order to simplify the problem, we employ the plane-parallel radiative transfer model with collimate irradiation to describe the phenomena.

Laser contribution in the governing bioheat transfer equation appears in the form of the divergence of radiative heat flux. It is given by ${ }^{(7)}$

$$
\frac{\partial q_{R, i}}{\partial x}=\frac{1}{V_{i}}\left(A_{i}^{R} \varepsilon_{i} E_{b, i}-\sum_{j=1}^{K} F_{j, i}^{A} Q_{R, j}\right)
$$

where for the element $i, V_{i}$ is volume, $A_{i}^{R}$ is the effective radiation area, $\varepsilon_{i}$ is emissivity, $E_{b, i}=n^{2} \sigma T_{i}^{4}$ is blackbody emissive power, $F_{j, i}^{A}$ is the absorption view factor and $Q_{R, j}$ is the radiant energy. $n$ is refractive index of biological tissue but the self emission term is negligible in this simulation.

The absorption view factor $F_{j, i}^{A}$ appearing in Eq. (2) is the fraction of the radiative 
energy leaving the radiation element $i$ which is absorbed by the radiation element $j$. It is related with the extinction view factor $F_{i, j}^{E}$ as

$$
F_{i, j}^{A}=\varepsilon_{j} F_{i, j}^{E}
$$

where the extinction view factor $F_{i, j}^{E}$ is computed from ${ }^{(7)}$

$$
F_{i, j}^{E}=\frac{1}{\pi A_{i}^{R}} \sum_{l=1}^{L}\left\{f_{i, j}\left(\mu_{l}\right) \mu_{l}\left[1-\exp \left(-\beta^{D} \Delta x_{i} / \mu_{l}\right)\right]\left[1-\exp \left(-\beta^{D} \Delta x_{j} / \mu_{l}\right)\right] \Omega_{l}\right\}
$$

where for a discrete direction having index $l, \mu=\cos \delta$ and $\Omega$ are the direction cosine and the weight, respectively, and $L$ is the total number of discrete directions over the polar space $0 \leq \delta \leq \pi$. In the present study, the discrete directions have been chosen according to the $\mathrm{S}_{8}$ quadrature set proposed by Fiveland ${ }^{(11)}$, and accordingly, values of ordinates $\mu$ and weights $\Omega$ have been taken from the literature. In Eq. (4), $f_{i, j}\left(\mu_{l}\right)$ is the fraction of the energy emitted from element $i$ in the direction having direction cosine $\mu_{l}$ which reaches element $j$. Considering that biological tissue is strong anisotropic scattering media, scaling function is introduced. The scaling is a widely used scheme for obtaining rapid yet accurate radiative transfer results for anisotropic scattering media. Guo and Maruyama ${ }^{(12)}$ have applied the zeroth-order delta function approximation using the $\mathrm{REM}^{2}$ method for the multi-dimensional media with quite good accordance with the exact solution.

$$
\beta^{D}=\beta\left(1-\omega a_{1} / 3\right), \quad \omega^{D}=\frac{\omega\left(1-a_{1} / 3\right)}{1-\omega a_{1} / 3},
$$

where $a_{l}$ is forward scattering parameter. Generally, a biological tissue is regarded as a quite strong forward scattering media, so that we assume $a_{1}=2.7$.

Considering contributions from all surface and volume elements, in Eq. (2), for any radiation element $i$, the radiative energy $Q_{R, i}$ is given by

$$
Q_{R, i}=A_{i}^{R} \varepsilon_{i} E_{b, i}+\sum_{j=1}^{K} F_{j, i} Q_{R, j}=A_{i}^{R} \varepsilon_{i} E_{b, i}+\sum_{j=1}^{K} F_{j, i}\left[\left(1-\omega_{j}^{D}\right) I_{b, j}+\omega_{j}^{D} I_{j}\right] A_{j}^{R}
$$

where $\omega$ is the scattering albedo, is the blackbody emission and for a given direction, $I$ is the average intensity in a radiation element. The effective radiation area $A_{i}^{R}$ is given by

$$
A_{i}^{R}=\sum_{l=1}^{L}\left[1-\exp \left(-\beta^{D} \Delta x_{i} / \mu_{l}\right)\right] \mu_{l} \Omega_{l}
$$

where $F_{j, i}$ appearing in Eq. (6) is related with $F_{j, i}^{E}$ as follows

$$
F_{j, i}=\omega_{i} F_{j, i}^{E}
$$

We present results in dimensionless form. In calculating the results, distance $x^{*}$, time $\xi$, temperature $\theta$, conductive heat flux $\Psi_{C}$ radiative heat flux $\Psi_{R}$, conduction-radiation parameter $N$, heat generation $g^{*}$ in the following way,

$$
x^{*}=\frac{x}{X}, \quad \xi=\frac{k t}{\rho c_{p} X^{2}}, \quad \theta=\frac{T}{T_{\text {ref }}},
$$




$$
\begin{array}{ccc}
N=\frac{k}{X \sigma T_{\mathrm{ref}}^{3}}, & \Psi_{C}=\frac{\partial \theta}{\partial x^{*}}, & \Psi_{R}=\frac{q_{R}}{N \sigma T_{\mathrm{ref}}^{4}}, \\
\Psi_{T}=\Psi_{C}+\Psi_{R}, & g_{\text {met }}^{*}=\frac{X^{2} q_{\text {met }}}{k T_{\mathrm{ref}}}, & g_{b}^{*}=\frac{X^{2} \rho_{b} c_{b} \gamma_{b}}{k} .
\end{array}
$$

Finally, the dimensionless form of bioheat transfer equation is expressed as

$$
\frac{\partial \theta}{\partial \xi}=\frac{\partial^{2} \theta}{\partial x^{* 2}}+g_{m e t}^{*}+g_{b}^{*}(1-\theta)-\frac{\partial \Psi_{R}}{\partial x^{*}} .
$$

\section{Physical Model}

Figure 1 shows the plane-parallel photon and heat transport model inside a biological tissue. The conduction part of bioheat transfer equation is solved by the finite volume method with implicit scheme. The boundary condition is as follows,

$$
\left.\frac{\partial \theta}{\partial x^{*}}\right|_{x^{*}=0}=0.0, \quad N \Psi_{\left.R\right|_{x^{*}=0}}=20.0, \quad \theta_{E}=1.0 .
$$

The initial temperature condition for biological tissue is set to $\theta=1.0$. The temperature in depth of the biological tissue is maintained at $\theta_{E}=1.0$. There is no heat loss from the surface, because, a worst situation for the biological tissue is considered. Generally, a continuous-wave laser power used for a laser therapy is around $1.0 \sim 10.0\left[\mathrm{~W} / \mathrm{cm}^{2}\right]$ so that a large incoming energy flux is considered. The metabolic heat production is negligible.

Considering that a biological tissue is strong anisotropic scattering medium for photon transport, the order of magnitude optical properties for biological tissue can be derived from the reference ${ }^{(13)}$. The objective of this study is to present a valuable guidance for laser therapy system, therefore, dimensionless results are presented here with varying these biological parameters. The thermophysical properties for human can be found in the references $^{(14-16)}$ Considering a variability of these properties, we assume that the biological parameters are; thermal conductivity, $k=0.3 \sim 0.6[\mathrm{~W} / \mathrm{mK}]$, blood perfusion rate, $\gamma_{b}=1.0 \sim 3.0 \times 10^{-3}\left[\mathrm{~m}^{3} /\left(\mathrm{s} \cdot \mathrm{m}^{3}\right)\right]$, extinction coefficient, $\beta=5.0 \sim 15.0\left[\mathrm{~mm}^{-1}\right]$, scattering albedo, $\omega=0.985 \sim 0.995$ for visible to near infrared light. Although this physical modeling is fundamental, the constructed simulation model can be also applied to pulsed laser therapy system in realistic computational condition.

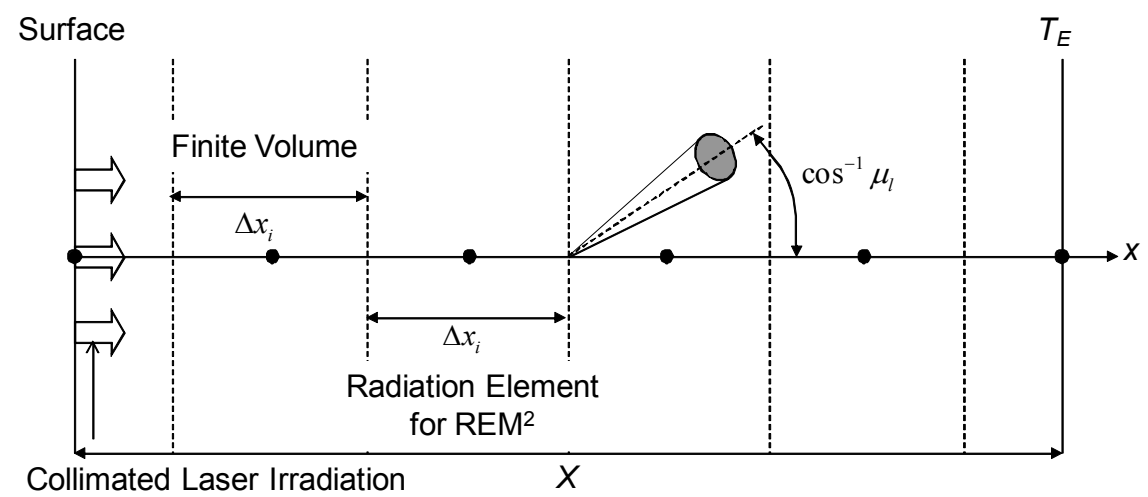

Fig. 1 plane-parallel photon and heat transport model inside a biological tissue 


\section{Results and Discussions}

\subsection{Effect of the extinction coefficient}

With $\omega=0.99, N=4.0$, and $g_{b}{ }^{*}=30.0$, and at 6 different time $\xi$ levels temperature $\theta$ distributions in the biological tissue have been compared with varying the extinction coefficient, namely, $\beta=5.0,10.0$ and $15.0\left[\mathrm{~mm}^{-1}\right]$, respectively in Figs. 2a-2c. For a given value of $\beta, \theta$ distributions have been plotted at $\xi=0.002,0.01,0.02,0.05,0.1$ and at the steady-state (SS). The dimensionless heat flux at steady-state is shown in Figs. 2d-2f. When extinction coefficient increases, the divergence of radiative heat flux becomes large, resulting in increasing the maximum temperature at the surface. As shown in the radiative

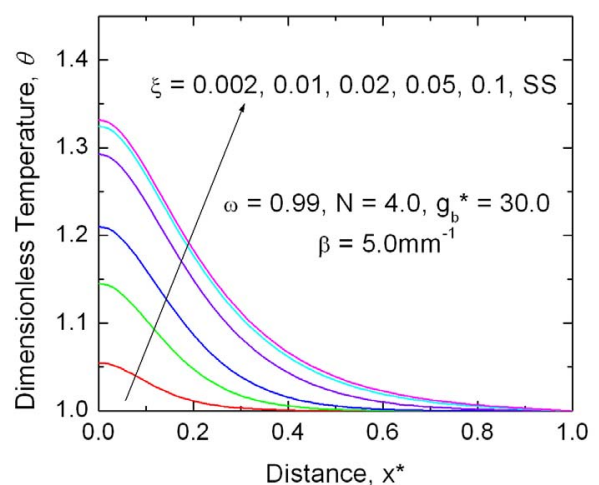

(a)

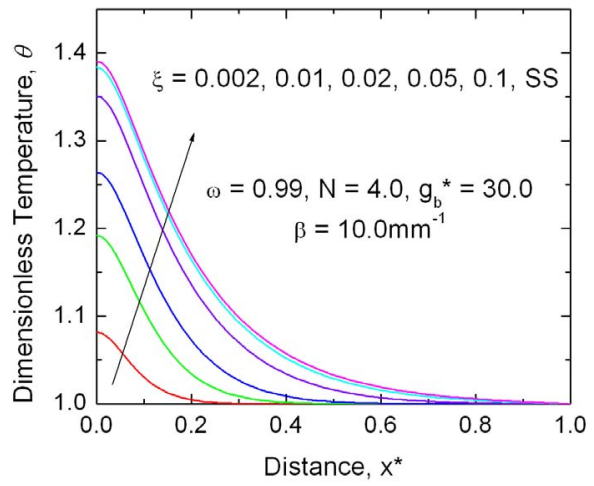

(b)

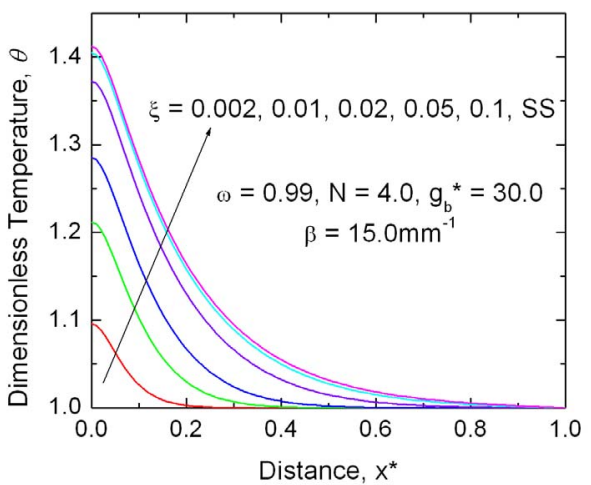

(c)

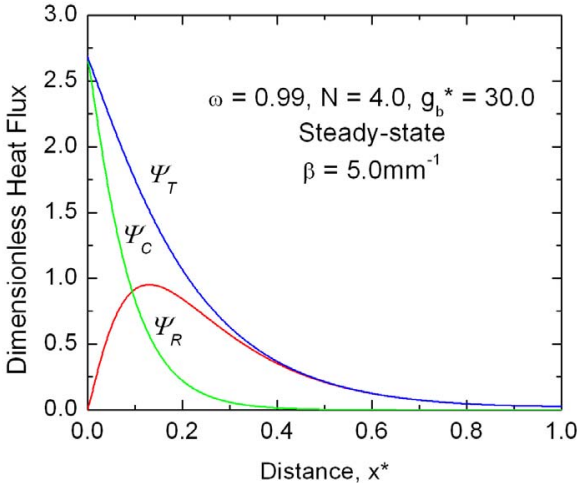

(d)

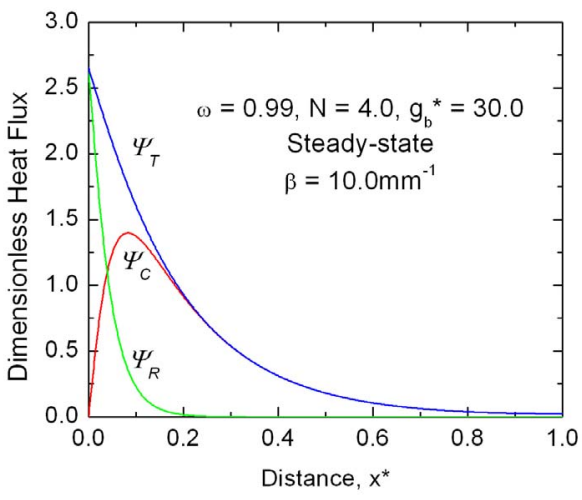

(e)

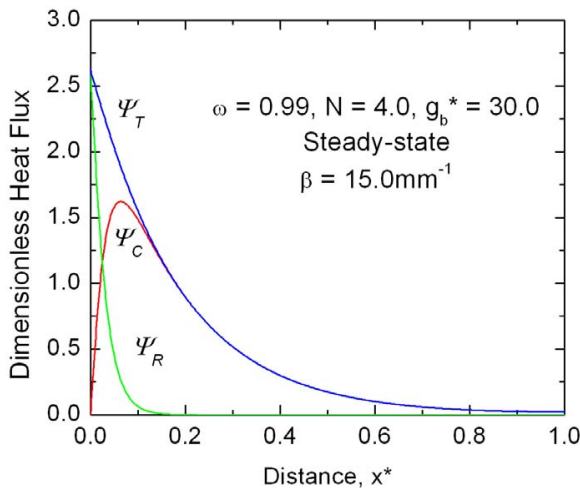

(f)

Fig. 2 Effect of extinction coefficient for dimensionless temperature distribution (a) (c) at different time levels and dimensionless heat flux (d) (f) at steady-state: (a, d) $\beta=5.0$ $\mathrm{mm}^{-1}$, (b, e) $\beta=10.0 \mathrm{~mm}^{-1}$, and (c, f) $\beta=15.0 \mathrm{~mm}^{-1} ; \omega=0.99, N=4.0, g_{b}^{*}=30.0$. 
heat flux distribution, the photon propagate depth becomes shallow because the absorption energy converted from laser power is more significant.

\subsection{Effect of the scattering albedo}

In Fig 3, the dimensionless temperature and the heat flux are presented for the effect of the scattering albedo with varying $\omega=0.985,0.99$ and 0.995 . With $\beta=10\left[\mathrm{~mm}^{-1}\right], N=4.0$, and $g_{b}{ }^{*}=30.0$, and at 6 different time $\xi$ levels temperature $\theta$ distributions in the biological tissue have been compared in Figs. 3a-3c. The dimensionless heat flux at steady-state is shown in Figs. 3d-3f. When scattering albedo increases, the scattered light increases, resulting in decreasing the maximum temperature at the surface.

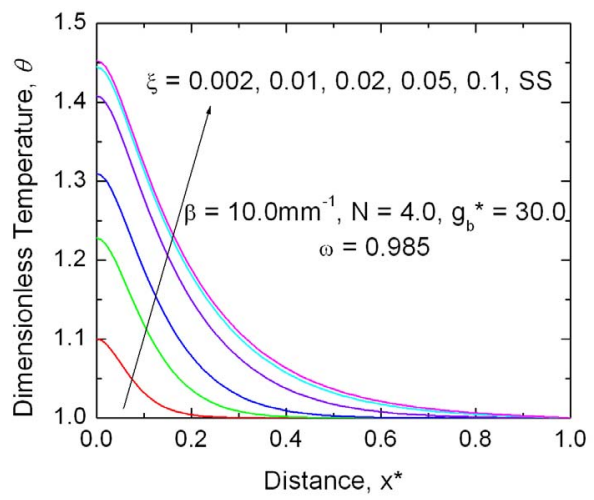

(a)

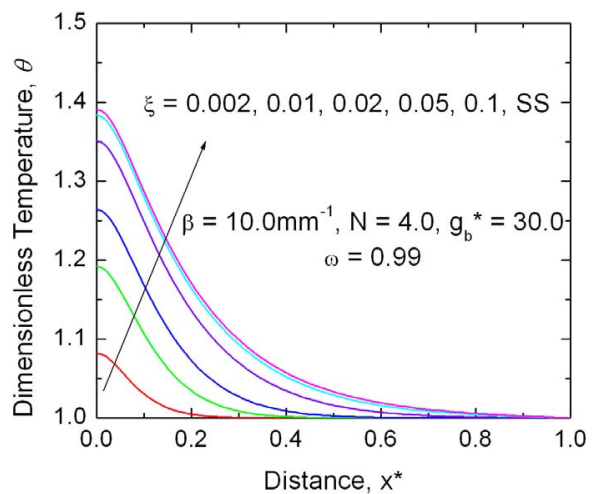

(b)

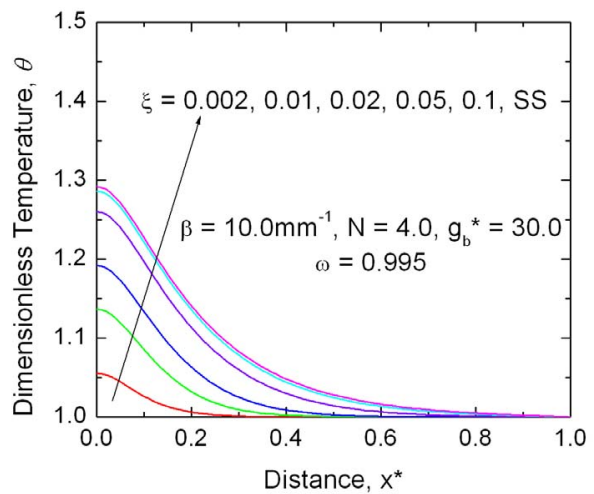

(c)

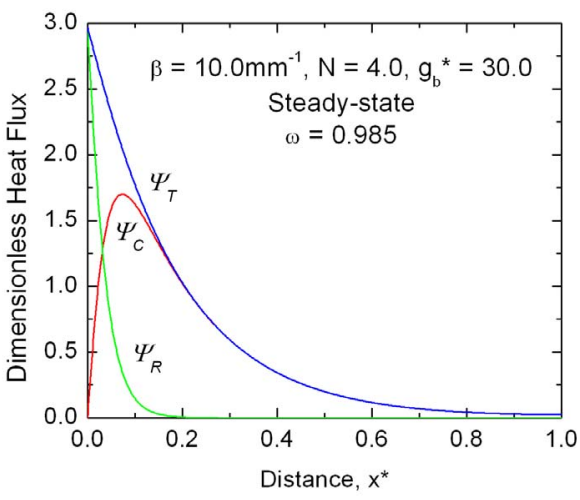

(d)

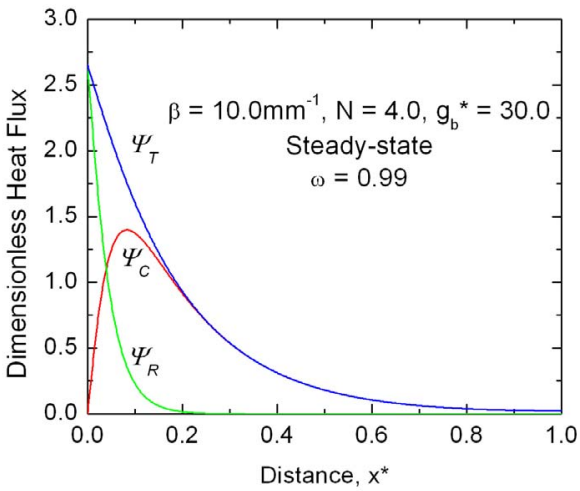

(e)

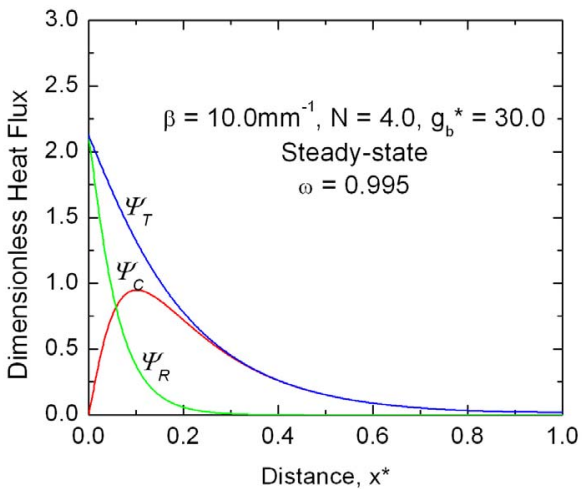

(f)

Fig. 3 Effect of scattering albedo for dimensionless temperature distribution (a) (c) at different time levels and dimensionless heat flux (d) (f) at steady-state: (a, d) $\omega=0.985$, (b, e) $\omega=0.99$, and (c, f) $\omega=0.995 ; \beta=10.0 \mathrm{~mm}^{-1}, N=4.0, g_{b}^{*}=30.0$. 
The dimensionless radiative heat flux also decreases, because the incident photons are scattered out to the surrounding air. The above-mentioned results indicate that optical properties of biological tissue are quite sensitive about temperature rise characteristics.

\subsection{Effect of the conduction-radiation parameter}

In Fig 4, dimensionless temperature and heat flux are presented for the effect of the conduction-radiation parameter with varying $N=4.0,6.0$ and 8.0 . With $\beta=10\left[\mathrm{~mm}^{-1}\right]$, $\omega=0.99$, and $g_{b}{ }^{*}=30.0$, and at 6 different time $\xi$ levels temperature $\theta$ distributions in the biological tissue have been compared in Figs. $4 \mathrm{a}-4 \mathrm{c}$. The dimensionless heat flux at

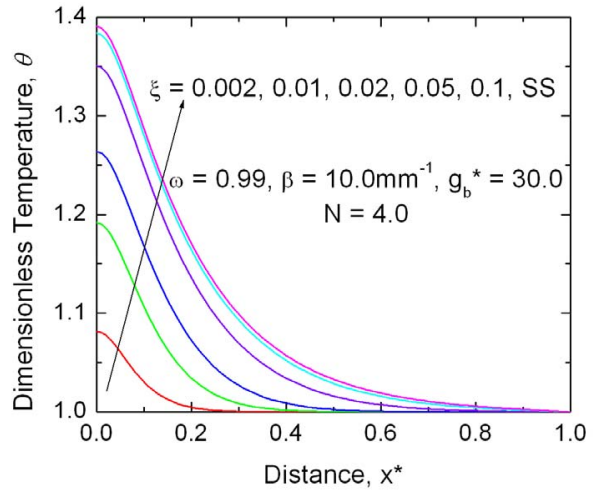

(a)

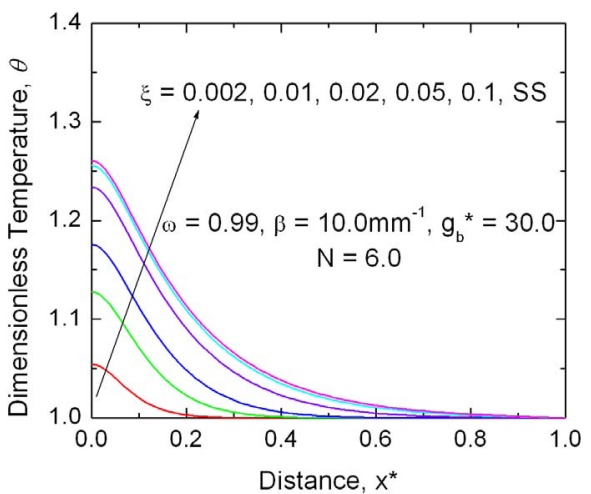

(b)

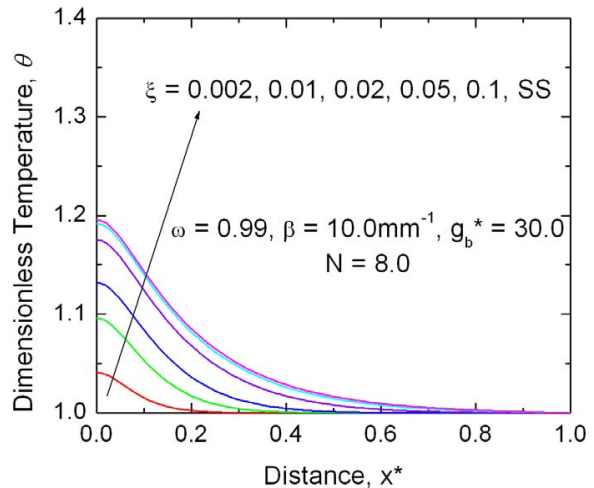

(c)

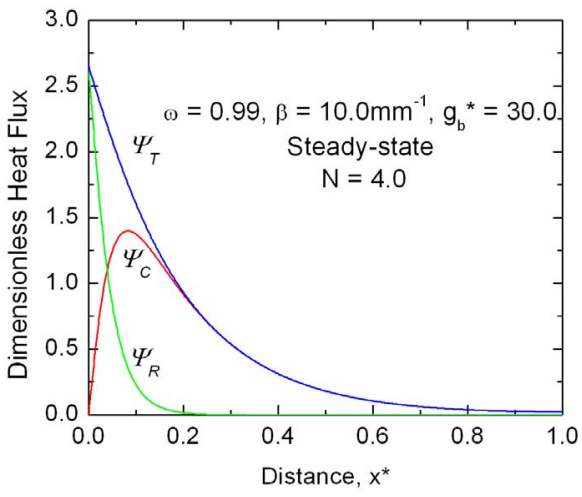

(d)

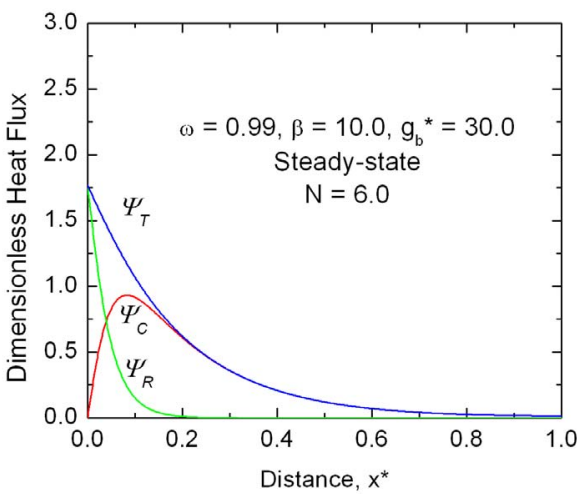

(e)

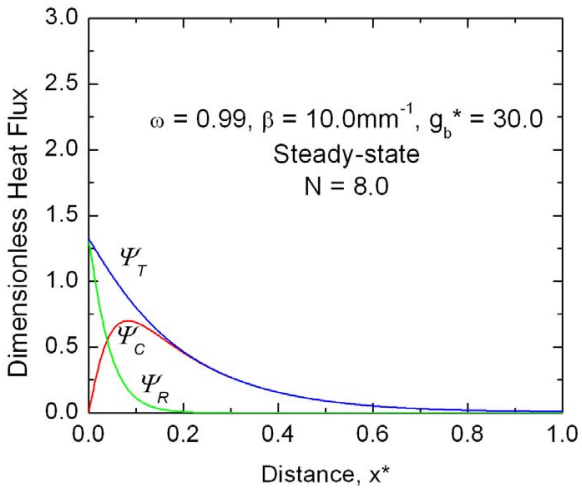

(f)

Fig. 4 Effect of conduction-radiation parameter for dimensionless temperature distribution (a) (c) at different time levels and dimensionless heat flux (d) (f) at steady-state: (a, d) $N=4.0$, (b, e) $N=6.0$, and (c, f) $N=8.0 ; \beta=10.0 \mathrm{~mm}^{-1}, \omega=0.99, g_{b}^{*}=30.0$. 
steady-state is shown in Figs. 4d-4f. When conduction-radiation parameter increases, the laser energy contribution is relatively insignificant. Namely, the generated heat is readily diffused with a high thermal conductivity. Therefore, the dimensionless temperature rise and dimensionless heat flux decrease with increasing $N$.

\subsection{Effect of the blood perfusion parameter}

In Fig 5, dimensionless temperature and heat flux are presented for the effect of the blood perfusion parameter with varying $g_{b}^{*}=30,60$ and 90 . With $\beta=10\left[\mathrm{~mm}^{-1}\right], \omega=0.99$, and $N=4$, and at 6 different time $\xi$ levels temperature $\theta$ distributions in the biological tissue have been compared in Fig. 5a-5c. The dimensionless heat flux at steady-state is

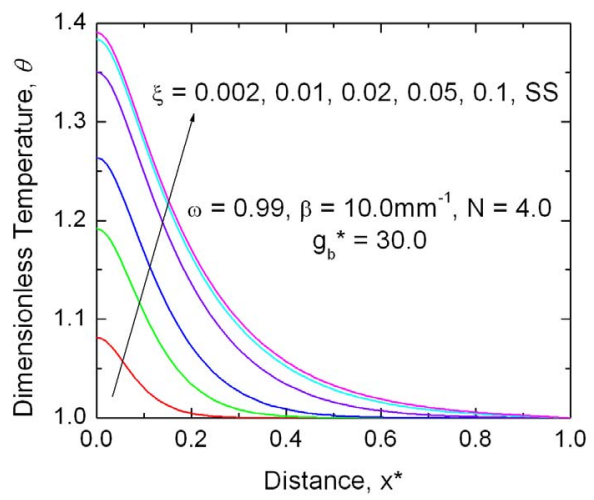

(a)

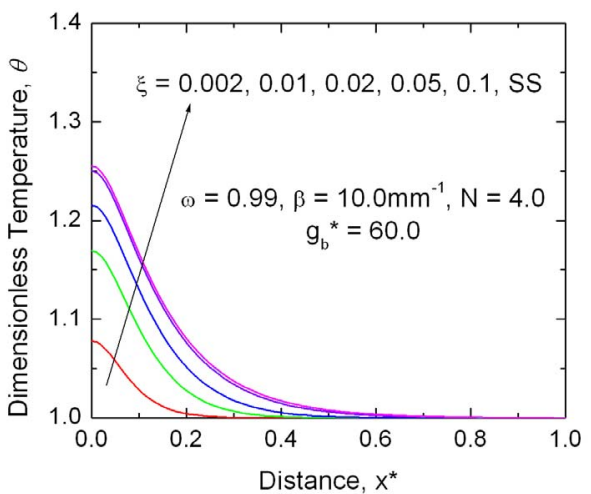

(b)

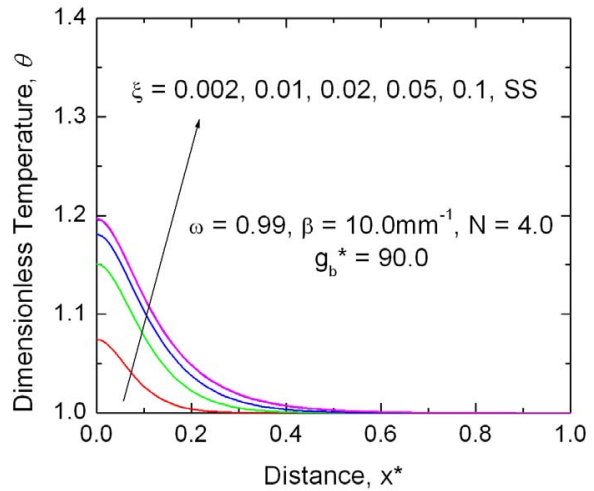

(c)

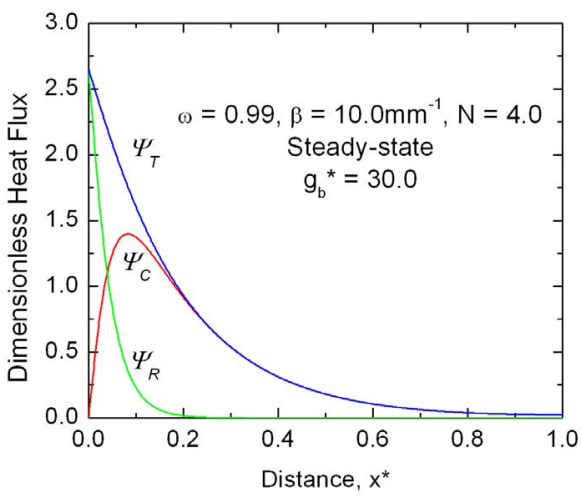

(d)

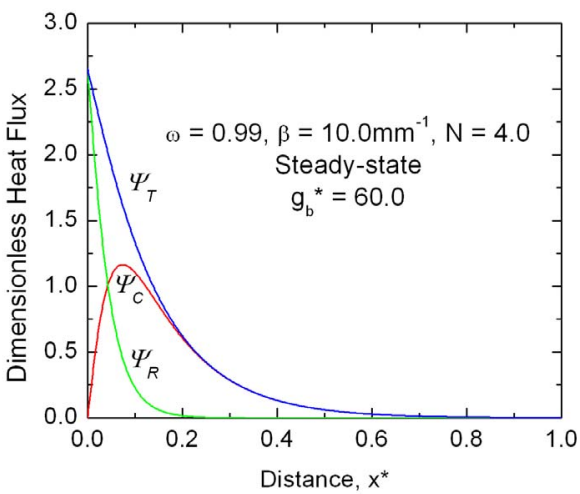

(e)

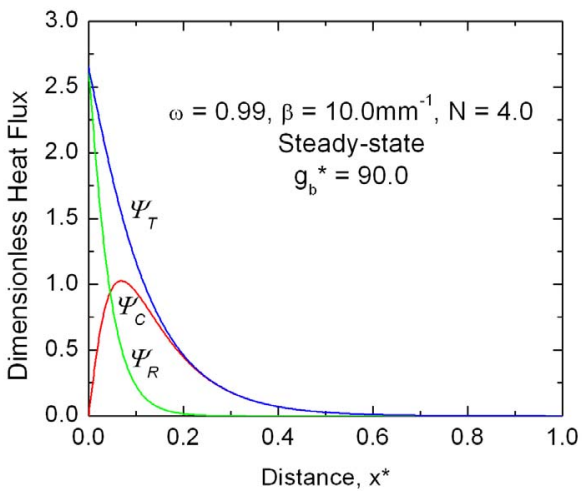

(f)

Fig. 5 Effect of blood perfusion parameter for dimensionless temperature distribution (a) (c) at different time levels and dimensionless heat flux (d) (f) at steady-state: (a, d) $g_{b}^{*}=30.0,(\mathrm{~b}, \mathrm{e}) g_{b}^{*}=60.0$, and (c, f) $g_{b}^{*}=90.0 ; \beta=10.0 \mathrm{~mm}^{-1}, N=4.0, \omega=0.99$. 
shown in Figs. 5d-5f. When blood perfusion parameter increases, the contribution of the heat transport by blood perfusion becomes significant. Namely, when a blood flow is active, the generated heat is more removed. Therefore, the dimensionless temperature rise of biological tissue decreases with increasing the blood perfusion parameter.

\subsection{Relation between the laser power and the irradiation time}

Therapists needs to know how much laser power and irradiation time are required for rising the biological tissue temperature up to $65^{\circ} \mathrm{C}$ (degeneration temperature of biological tissue) with different depth. This section demonstrates the diagram for the relation between the laser power and the irradiation time in Fig. 6. The thermophysical properties and optical properties are set to; thermal conductivity, $k=0.45[\mathrm{~W} / \mathrm{mK}]$, blood perfusion rate, $\gamma_{b}=1.3 \times 10^{-3}\left[\mathrm{~m}^{3} /\left(\mathrm{s} \cdot \mathrm{m}^{3}\right)\right]$, extinction coefficient, $\beta=10.0\left[\mathrm{~mm}^{-1}\right]$, scattering albedo, $\omega=0.99$. The laser power is changed from 1 to $10\left[\mathrm{~W} / \mathrm{cm}^{2}\right]$. The initial temperature of biological tissue is $37^{\circ} \mathrm{C}$ and the maximum biological tissue depth is $50[\mathrm{~mm}]$. In Fig. 6, the three different depths are shown at 0, 2.5, 5, 7.5 and $10[\mathrm{~mm}]$. When laser power increases, the irradiation time to $65^{\circ} \mathrm{C}$ decreases at all depths. The irradiation time to $65^{\circ} \mathrm{C}$ for deep part is quite longer than that for the surface.

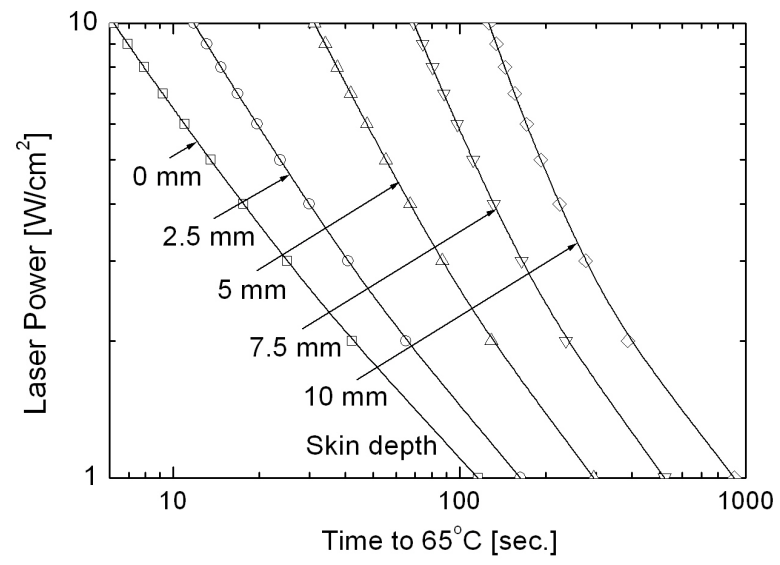

Fig. 6 Relation between the laser power and the irradiation time to $65^{\circ} \mathrm{C}$ (degeneration temperature of biological tissue), with different depth.

\section{Conclusion}

The present paper has been described the new simulator for laser therapy. In order to predict the photon and heat transport characteristics inside a biological tissue, the photon transport is solved with the REM ${ }^{2}$, and then coupled with bioheat transfer equation. Conclusions are summarized as:

1. We have demonstrated the effect of optical properties of biological tissue with varying extinction coefficient and scattering albedo. The temperature distribution inside a tissue has been changed with slightly different optical properties. The results indicate that optical properties of biological tissue are quite sensitive to temperature rise characteristics.

2. The effect of thermophysical properties has been investigated. When laser energy contribution is insignificant compared with the heat conduction, the temperature rise was decreased. When a blood flow is active, the generated heat is more removed. Therefore, the dimensionless temperature rise decreases with increasing the blood perfusion rate.

3. We have demonstrated the diagram for the relation between the laser power and the irradiation time. The diagram allows readers to know how much laser power and irradiation time are required for rising the biological tissue temperature up to $65^{\circ} \mathrm{C}$ (degeneration temperature of biological tissue). 


\section{Acknowledgement}

This research was supported by Grant for Promotion of Niigata University Research Projects B. The authors acknowledge Prof. Subhash C. Mishra at Indian Institute of Technology Guwahati and Mr. Naoya Ogasawara at Tohoku University for their valuable advice.

\section{References}

(1) Jaunich M, Raje S, Kim K, Mitra K, Guo Z, Bio-heat transfer analysis during short pulse laser irradiation of tissues, International Journal of Heat and Mass Transfer, 51 (2008) 5511-5521.

(2) Nitta I, Zhao X, Kanno A, Kan Y, Yoshimasa T, Maruyama T, Maeda Y, Laser treatment of cutaneous lesions with image-guided fine spot-scanning irradiation, Proc. SPIE, vol. 6826, (2007) 68260H.

(3) Panjehpour M, Wilke A, Frazier DL, Overholt BF, Hyperthermia treatment using a computer controlled Nd:YAG laser system in combination with surface cooling, Proc. SPIE (1991) 307-315.

(4) Eda H, Oda I, Ito Y, Wada Y, Oikawa Y, Tsunazawa Y, Takada M, Tsuchiya Y, Yamashita Y, Oda M, Sassaroli A, Yamada Y, Tamura M, Multichannel time-resolved optical tomographic imaging system, Rev. Sci. Instrum. 70 (1999) 3595.

(5) Arimoto H, Egawa M and Yamada Y, Depth profile of diffuse reflectance near-infrared spectroscopy for measurement of water content in skin, Skin Research and Technology, 11(2005) 27-35.

(6) Yamada J, Kawamura A, Miura Y, Takata S, Ogawa K, Study on radiation transfer in human skin for cosmetics, J. Quant. Spectrosc. Radiat. Transf. 93 (1-3)(2005) 219-230.

(7) Maruyama S, Light Energy Engineering, Yoken-do, Tokyo.(2004)(In Japanese)

(8) Maruyama S, Aihara T, Radiation heat transfer of arbitrary three-dimensional absorbing, emitting and scattering media and specular and diffuse surfaces, J. Heat Transf--Trans. ASME, 119 (1997) 129-136.

(9) Maruyama S, Guo ZX, Radiative heat transfer in arbitrary configurations with nongray absorbing, emitting, and anisotropic scattering media, J. Heat Transf-Trans. ASME, 121 (1999) 722-726.

(10) Pennes H, Analysis of tissue and arterial blood temperatures in the resting human forearm, J. Appl Physiol, (1949)93-122.

(11) Fiveland WA, Jessee JP, Comparison of discrete ordinates formulations for radiative heat-transfer in multidimensional geometries, J. Thermophys. Heat Transf. 9 (1)(1995)47-54.

(12) Guo ZX, Maruyama S, Scaling anisotropic scattering in radiative transfer in three-dimensional nonhomogeneous media, Int. J. Heat Mass Transf. 26,(1999) 997-1007.

(13) Bashkatov AN, Genina EA, Kochubey VI, Tuchin VV, Optical properties of human skin, subcutaneous and mucous tissues in the wavelength range from 400 to $2000 \mathrm{~nm}$ Journal of Physics D-Applied Physics, 38(2005): 2543-2555.

(14) Jiang SC, Ma HJ, Li HJ, Zhang XX, Effect of thermal properties and geometrical dimensions on skin burn injuries, Burns, 28(2002)713-717.

(15) Brix G, Martin S, Gesine H, Jürgen G, Estimation of heat transfer and temperature rise in partial-body regions during MR procedures: An analytical approach with respect to safety considerations, Magn Reson Imaging, 20(2002)65-76.

(16) Maruyama S, Okajima J, Komiya A, Takeda H, Estimation of temperature distribution in biological tissue by using solutions of bioheat transfer equation, Heat Transfer Asian Research, 37(2008) $374-386$. 